\title{
IGUALDAD DE GÉNERO Y DIVERSIDAD SEXUAL: PERCEPCIÓN DE PADRES/MADRES Y RESPONSABLES DE NIÑOS Y NIÑAS DE UNA ESCUELA DE MACAPÁ-AP
}

\author{
Igualdade de gênero e diversidade sexual: percepção de pais/mães e responsáveis \\ de crianças de uma escola de Macapá-AP
}

\section{Gender equality and sexual diversity: perception of parents and guardians of children of a school in Macapá-AP}

Alexandre Adalberto Pereira ${ }^{1}$

Andrés Lema Mendez ${ }^{2}$

\begin{abstract}
Resumen
Este artículo presenta el análisis de las respuestas del cuestionario realizado a padres, madres y responsables participantes de la reunión que sucedió en una escuela de la ciudad de Macapá, Amapá Brasil en la cual se desenvuelve el proyecto de maestría que aborda la temática igualdad de género y diversidad sexual. Utilizamos los aportes teóricos de Sorj (2014), Madeiros; Pinheiros (2013), y Andrade (2015) que posibilitan entender los datos de las percepciones arrojados sobre igualdad de género. Algebaile (2017), Ratier (2016), Manhas (2016), Ximenes (2016) y Cara (2016) como sustentos para análisis en lo competente a diversidad sexual. Concluimos que los encuestados tienden a asumir respuestas indiferentes con respecto al tema de la diversidad sexual y la igualdad de género y que el abordaje de las mismas en la esfera educacional resulta relevante a la hora de generar estrategias para reducir los casos de violencia, opresión, y LGBTfobia. El proyecto cuenta con financiación (becas) por el Programa de Alianzas para la Educación y la Capacitación (Becas Brasil - PAEC OEA-GCUB).
\end{abstract}

PALABRAS CLAVE: Igualdad de género. Diversidad sexual. Educación.

\footnotetext{
${ }^{1}$ Doutor em Educação pelo Programa de Pós-Graduação em Educação da Universidade Federal de Uberlândia (2013). Mestre em Cultura Visual pela Universidade Federal de Goiás). Licenciatura Plena em Artes Visuais pela Universidade Federal de Goiás . Professor do Curso de Licenciatura em Artes Visuais e do Programa de Pós-Graduação em Educação da Universidade Federal do Amapá-AP. Endereço: Universidade Federal do Amapá, Reitoria, Colegiado de Artes. Rodovia Jucelino Kubscheck de Oliveira Km 02 Zerão, 68900000 - Macapá, AP/ Brasil. Contato: (96) 32415070. E-mail: pereiraxnd@gmail.com Orcid: https://orcid.org/0000-0003-4029-1415

${ }^{2}$ Graduação em Serviço Social pela Universidad de la Republica Uruguay. Mestrado em Educação pelo Programa de Pós-graduação em Educação da Universidade Federal de Amapá (PPGED/UNIFAP). Realiza mobilidade acadêmica no Programa de Pos-graduação em Estudos Interdisciplinares sobre Mulheres, Gênero e Feminismo (PPGNEIM) da Universidade Federal da Bahia (UFBA). Endereço: Universidade Federal do Amapá, Rodovia Juscelino Kubitschek - de 1670/1671 (ao fim Universidade) 68903419 - Macapá, AP/Brasil. Contato: (55) 9198535418. E-mail: andreslemamendez@gmail.com Orcid: https://orcid.org/0000-0001-5959-5799
} 


\title{
Resumo
}

Esse artigo apresenta um recorte do projeto de mestrado que aborda a temática da igualdade e gênero e diversidade sexual em contexto escolar na cidade de Macapá, Amapá, Brasil. Analisamos respostas de um questionário aplicado a pais, mães e responsáveis que participaram de uma reunião na escola onde foi apresentado o referido projeto de investigação. Utilizamos os aportes teóricos de Sorj (2014), Madeiros; Pinheiros (2013) e Andrade (2015) para entender os dados sobre as percepções sobre igualdade de gênero. Algebaile (2017), Ratier (2016), Manhas (2016), Ximenes (2016) e Cara (2016) sustenta a análise do componente diversidade sexual. Concluímos que os colaboradores tendem a assumir respostas indiferentes com relação ao tema da diversidade sexual e de gênero e que a abordagem sobre o tema na esfera educacional tornase relevante para se pensar em estratégias que reduzam a violência, opressão e LGBTfobia. O projeto conta com financiamento (bolsa de estudos) pelo Programa de Alianças para a Educação e a Capacitação (Bolsas Brasil - PAEC OEA-GCUB).

PALAVRAS-CHAVE: Igualdade de gênero. Diversidade sexual. Educação.

\begin{abstract}
This article presents an excerpt from the master's project that focuses on the theme of equality and gender and sexual diversity in a school context in the city of Macapá, Amapá, Brazil. Data from a questionnaire applied to parents and guardians who participated in a meeting at the school where the referred research project was presented are analyzed. We used the theoretical contributions of Sorj (2014), Madeiros; Pinheiros (2013) and Andrade (2015) to understand the perceptions about gender equality arising from the data. Algebaile (2017), Ratier (2016), Manhas (2016), Ximenes (2016) and Cara (2016) support the analysis of the sexual diversity component. We conclude that the research collaborators, with respect to the theme of sexual and gender diversity, tend to assume indifferent answers. We understand that discussing the theme in education is relevant to think about strategies that reduce violence, oppression and LGBTphobia. The project is sponsored by the Alliance for Education and Training Program (Scholarships Brazil - PAEC OEA-GCUB).
\end{abstract}

KEYWORDS: Gender equality. Sexual diversity. Education.

\section{INTRODUCCIÓN}

El presente artículo busca exponer el trabajo que se ha venido desarrollando a partir de la aprobación por parte de quien suscribe en el "Programa de Alianças para a Educação e a Capacitação" (Bolsas Brasil-PAEC OEA-GCUB) ${ }^{3}$, y la ejecución del proyecto de investigación inicialmente propuesto.

\footnotetext{
${ }^{3}$ Dicha beca tiene los objetivos de promover la democracia, defender los derechos humanos, garantir un abordaje multidimensional para la seguridad, promover el desarrollo integral y la prosperidad, y apoyar la cooperación jurídica interamericana. La misma es una iniciativa de la Organización de los Estados Americanos (OEA), y el Grupo Coimbra de Universidades Brasileñas (GCUB) con el apoyo de la División de Temas Educativos de Relaciones Exteriores de Brasil (MRE) y la Organización Panamericana de la Salud (OPS/OMS). Asimismo, dicha beca apoya a los Estados Miembros en la creación y ejecución de programas que promueven el desarrollo humano en todos los niveles educativos, además de buscar fortalecer los valores democráticos y de seguridad, buscando la integración regional y
} 
Cabe hacer mención entonces a lo que fue inicialmente el proyecto en la etapa de postulación, el cual buscaba investigar la relación entre la comunidad educativa uruguaya con una organización sin fines de lucro que se encuentra interviniendo en las escuelas públicas de Montevideo, abordando la temática de la igualdad de género y la diversidad sexual. Sería entonces que a la luz de analizar el impacto en la comunidad educativa de la intervención realizada por esta organización sin fines de lucro existente en Uruguay, llamada GEDUCA ${ }^{4}$, que imparte talleres lúdicos-recreativos en escuelas públicas de Montevideo, en lo que a través del juego, visualizan la existencia de roles estereotipados y propician la deconstrucción de los mismos desde la propia vivencia que sucede en el taller, abordando de esta forma la igualdad de género y el respeto a la diversidad sexual.

Por lo tanto, inicialmente el proyecto de investigación tenía como objetivo analizar el impacto que tiene en la comunidad educativa uruguaya los talleres lúdicosrecreativos generados por GEDUCA en torno a los conceptos de igualdad de género y diversidad sexual. Para ello, se proponía realizar observación participante en los talleres que se imparten en las escuelas, buscando rescatar las intervenciones de los niños y las niñas participantes, como también realizar entrevistas semiestructuradas a informantes calificados de la comunidad educativa. Resulta relevante realizar esta contextualización de lo que se proponía inicialmente en el proyecto de investigación, debido a que este era el punto de partida inicial, y a partir de la aprobación del mismo, y la puesta en ejecución durante el primer año de maestría ya cursado, el mismo fue adquiriendo modificaciones que se expondrán a continuación.

El proyecto fue aprobado para cursarse en la ciudad de Macapá, en el estado de Amapá, en la región amazónica del norte de Brasil, en la Universidad Federal de Amapá. Cuando se llega allí, el proyecto es presentado en varias oportunidades, inicialmente en las disciplinas de "Seminário de Dissertação", donde se discuten los objetos de investigación. Además, también se presentó el proyecto en el grupo de investigación del que se forma parte ${ }^{5}$.

Asimismo, se es realizado el estado del arte de la investigación en Brasil, con el objetivo de comprender el objeto de investigación considerando lo que ya se encuentra producido sobre la temática de la igualdad de género y la diversidad sexual en relación con la infancia a nivel nacional, pero particularmente en la zona norte de Brasil. Es a partir de la realización de este estado del arte que se percibe que hay escasa y casi nula producción sobre la temática en la zona norte del país.

Es entonces que es propuesto el desafío investigativo de realizar talleres lúdicosrecreativos en una comunidad educativa de Macapá, especialmente en la escuela pública, a fin de trabajar con niños y niñas sobre la temática de la igualdad de género y la diversidad sexual en la zona norte del Brasil. Cabe mencionar aquí, que es realizado una reunión con padres, madres y responsables de los niños y las niñas que participarían de la investigación, con el objetivo de presentar el proyecto de investigación, solicitar la firma de los términos de autorización, y realizar un cuestionario a fin de comprender la

fomentando oportunidades de formación para los ciudadanos miembros de los Estados Miembros a fin de mejorar la calidad y equidad en la educación.

${ }^{4}$ La misma es una Cooperativa, que inicialmente fue una ONG, que posee un equipo multidisciplinario con compromiso por la igualdad de género y el respeto a la diversidad sexual. Dicha cooperativa trabaja por la defensa de los derechos sexuales y reproductivos, desde el ámbito educativo en Uruguay. Su sitio web es: https://geducauy.wixsite.com/somosgeduca

${ }^{5}$ Grupo de Pesquisa Educação, Decolonialidade e Diversidades. 
percepción sobre la temática de la igualdad de género y la diversidad sexual de acuerdo a la visión de los padres, madres y representantes de los niños y las niñas que participarían de la investigación. Por lo tanto, actualmente la investigación tomo otros rumbos, la primera adecuación fue llevar el trabajo de campo a la ciudad de Macapá, en el estado de Amapá en la región Amazónica de Brasil, en una escuela pública de dicha ciudad. El problema que se busca responder ahora con la aplicación del mismo es ¿Cuál será el impacto generado por los talleres lúdicos-recreativos que abordaran la cuestión de la igualdad de género y la diversidad sexual en el contexto escolar de una escuela de Macapá, Amapá, Brasil?

Por lo tanto, el objetivo general de este artículo es analizar el proceso de implementación del proyecto de maestría que aborda la temática de la igualdad de género y la diversidad sexual en el contexto escolar de la ciudad de Macapá, Amapá, Brasil. Como objetivos específicos para el presente artículo se propone indagar las respuestas del cuestionario realizado a padres, madres y responsables participantes de la reunión realizada en la escuela en la cual se presenta el proyecto de investigación y el material didáctico que se utilizara en los talleres lúdicos-recreativos.

El problema que se busca responder durante la realización del artículo es identificar qué tipo de opiniones poseen los padres, madres y representantes de los niños y las niñas en lo competente a la educación de la igualdad de género y la diversidad sexual.

El presente artículo describirá entonces el análisis realizado a partir de las respuestas del cuestionario de los padres, madres y representantes que participaron de la reunión donde se presentó el proyecto de investigación en la comunidad educativa de la ciudad de Macapá.

Finalmente, se menciona que el artículo se relaciona con la temática sugerida por la revista, ya que el mismo aborda la temática de la igualdad de género y la diversidad sexual, temática que ha sido tratada de ser excluida de la educación básica brasilera, por parte de diferentes movimientos conservadores, tales como "Escolas sem Partido".

Además, se plantea que el presente proyecto al buscar abordar la cuestión de la igualdad de género y la diversidad sexual en el contexto escolar, buscará problematizar la sociedad actual, basada en la jerarquía entre hombres y mujeres, lo que aparece como un problema para alcanzar la igualdad de género, además de trabajar la diversidad sexual en sala de aula, buscando problematizar las situaciones de machismo/misoginia y LGBTfobia en el contexto escolar, teniendo en vista la dificultad en el abordaje de esas cuestiones en el contexto educacional, resultante del conservadurismo de la sociedad brasilera.

\section{Resultados y discusiones}

En la presente sección trabajaremos analizando las respuestas que los padres, madres y responsables de los niños y las niñas que acudieron a la reunión donde se presentó el proyecto de investigación, el material didáctico que se utilizará durante los talleres lúdicos-recreativos y se solicitó las autorizaciones para realizar los mismos. Vale aclarar que de los 100 padres, madres y responsables que fueron convocados, acudieron 22 padres, madres y responsables de niños y de niñas, pero solo 15 de los mismos respondieron los cuestionarios. 


\section{Percepción de los padres/madres y responsables de los niños y las niñas sobre Igualdad de Género y Diversidad Sexual}

En cuanto al sexo de los que respondieron al cuestionario, se visualiza una clara presencia de mujeres, siendo un $66.7 \%$ mujeres, y un $33.3 \%$ de hombres. Respecto al vínculo de parentesco que presentan con los niños y las niñas, se visualiza que un $60 \%$ de los participantes son madres del alumno/a, un $26.7 \%$ son padres del alumno/a, y un $13.3 \%$ responsables por el/la alumno/a, en una amplia mayoría siendo estos abuelos/as, $\mathrm{y}$ en menor proporción madrastras.

En cuanto al lugar de nacimiento de quienes respondieron al cuestionario, se encuentra una clara presencia de la zona norte de Brasil, teniendo una presencia mayor del estado de Pará principalmente, de la ciudad de Belém. En una menor proporción se encuentran los de origen del estado de Amapá, la ciudad de Macapá, y solo hay una persona que pertenece a la región del nordeste, siendo del estado de Maranhão.

En lo que hace referencia a la descendencia étnico-racial de los y las participantes, un $80 \%$ se considera de descendencia "pardo/a", y un $20 \%$ de descendencia "blanco/a".

En lo que respecta a la religión, hay una proporción del 33.3\% de católicos/as, y un $33.3 \%$ también de evangélicos/as. Hay un $13.3 \%$ de católicos/as no practicantes, y esta misma proporción de personas que creen en dios, pero no siguen ninguna religión. Finalmente, hay un $6.7 \%$ de personas que prefieren no declarar.

Por otra parte, se realizaron dos preguntas las cuales tienen que ver con cuál es la actividad con la que ocupan más tiempo, y cuál es el medio que utilizan para mantenerse informados sobre los acontecimientos actuales, siendo la respuesta "televisión" en el caso de la pregunta uno (con un 66.7\%), y "jornal falado" en la pregunta dos (con un 54.4\%), seguido por "internet" en la pregunta uno (13.3\%), y la misma respuesta para la pregunta dos con un 33.3\%. En cuanto al medio que mas utilizan para formar opiniones y percepciones, los padres, madres y representantes indicaron el "jornal falado (TV)", con un $40 \%$, seguido por "igreja", con un $20 \%$, e "internet" con un 13.3\%. En lo que respecta a sobre en cual medio han escuchado hablar sobre "igualdad de género", los/as encuestados/as responden en un $60 \%$ que han escuchado hablar en "Jornal falado (TV)", y un 13.3\% en "Escola", e "Internet" consecutivamente. En cuanto a cuál es el medio donde escucharon hablar sobre diversidad sexual, un $35.7 \%$ expresa que escucho hablar sobre ello en "Jornal falado (TV)", un $28.6 \%$ "Internet", y un $14.3 \%$ en la "Escola".

Se puede visualizar de esta forma, que en lo referente a la igualdad de genero las personas comentan que han escuchado hablar sobre ello en "jornal falado" (60\%) mientras que en la diversidad sexual dicho porcentaje desciende (35.7\%), lo que demuestra de esta forma que en los medios de comunicación interfieren creando significaciones y percepciones sobre la igualdad de género. A medida que avancemos durante el artículo, profundizaremos sobre cómo se configura este rol.

Respecto al nivel de escolaridad de sus padres, un $33.3 \%$ declara que su padre curso hasta " $1{ }^{\circ}$ a $5^{\circ}$ ano de Ensino Fundamental (antiguo primário)", seguido por un $26.7 \%$ de "no estudo". Con respecto al nivel educativo de sus madres, un $33.3 \%$ declaran que su madre "no estudo", seguido por un $26.7 \%$ de "Ensino Medio" y " $1{ }^{\circ}$ a $5^{\circ}$ ano de Ensino Fundamental (antiguo primário)" consecutivamente. Finalmente, en lo 
que respecta al propio nivel educativo de quienes respondieron el cuestionario, un $40 \%$ declara "Ensino Medio", seguido por un 26.7\% de "Especialização".

En lo que respecta al ingreso familiar mensual, un $40 \%$ declara "De 1 a 3 salarios mínimos", y también un 40\% declara "Ate 1 salario mínimo". Solo el 13.3\% señalo tener de ingresos "De 3 a 6 salarios mínimos".

Ahora bien, adentrándonos en sus percepciones sobre lo que comprenden por igualdad de género resulta relevante traer a colación la siguiente gráfica, que demuestra sus percepciones sobre lo que ellos/as creen sobre la cantidad de horas que trabajan las mujeres:

Gráfica 1- Percepción, por parte de los padres y madres de los alumnos, sobre la igualdad de género

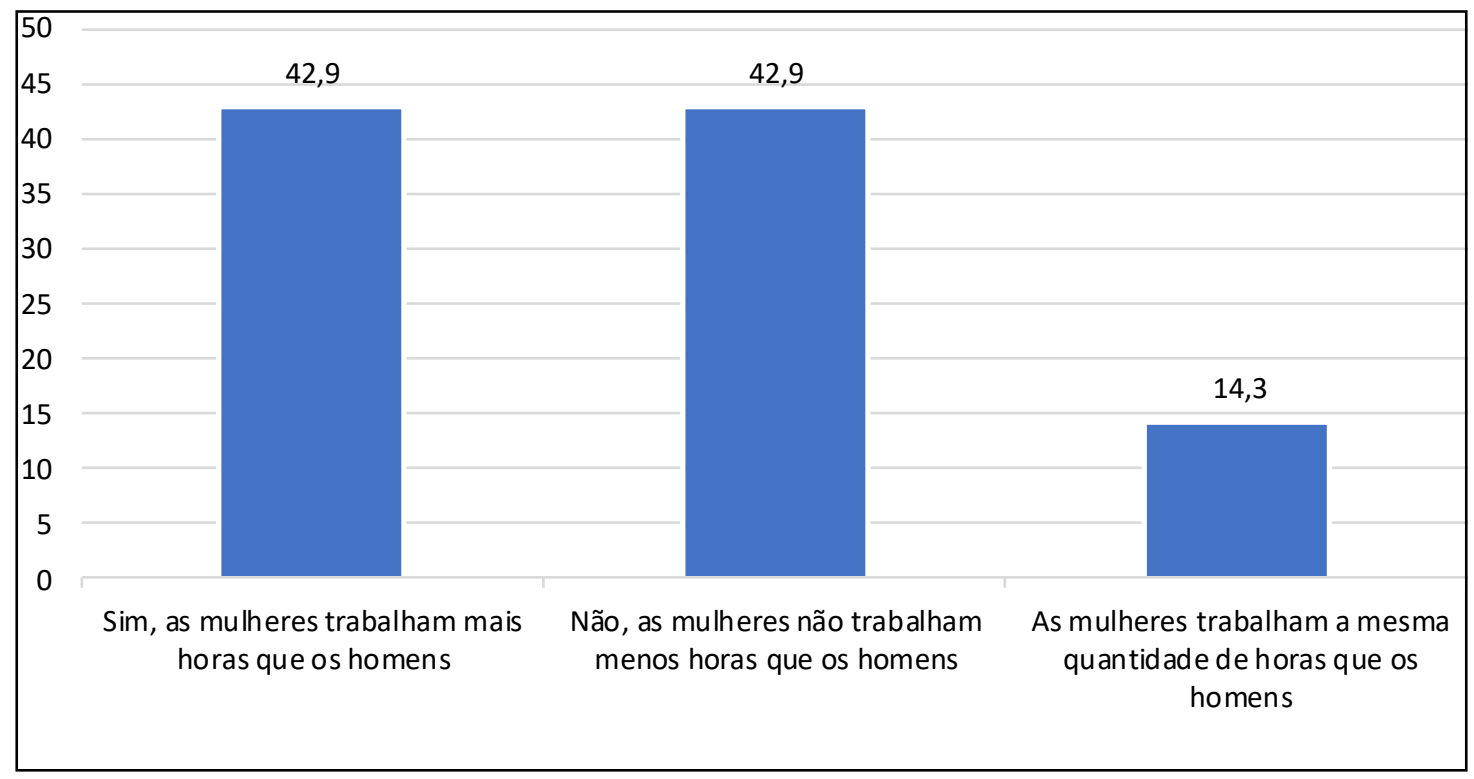

Fonte da pesquisa dos autores.

En cuanto a la cantidad de horas trabajadas por mujeres, es conveniente aquí citar a Sorj, quien expresa que "[...] mães com filhos dependentes dedicam, em média, o triplo de horas aos afazeres domésticos" (SORJ, 2014, p. 126). Ello se encuentra sobreentendido cuando el $42.9 \%$ de quienes respondieron que perciben que las mujeres trabajan más horas que los hombres. Asimismo, resultan relevantes los aportes de Medeiros y Pinheiro (2018) que comentan "A divisão sexual do trabalho não é caracterizada por um espelhamento de atividades e, por isso, as mulheres invariavelmente trabalham mais que os homens" (MEDEIROS; PINHEIRO, 2018, p. 161). De este modo, podemos compreender que

trabalho profissional e trabalho doméstico, produção e reprodução, assalariamento e família, classes sociais e sexo social são categorias indissociáveis. $\mathrm{O}$ trabalho reservado às mulheres na esfera produtiva, bem como a sua precarização e baixa remuneração, reflete sua posição na hierarquia de gênero existente tanto na família quanto no mundo do trabalho. Nesse sentido, produção e reprodução condicionam-se reciprocamente, alimentando um padrão de divisão do trabalho que garante a reprodução de todo o sistema social. Essa divisão sexual do trabalho contribui, igualmente, 
para generalizar e acentuar o processo de precarização do trabalho, que se torna cada vez mais "feminino" em sua caracterização - isto é, com a predominância de ocupações subordinadas que exigem baixa qualificação, pagam baixos salários e são realizadas em jornadas parciais. (ANDRADE, 2015, p. 290).

Además, es clara la existencia de una división sexual del trabajo, y en esta división la mayor responsabilidad por el trabajo doméstico es femenina. Respecto a esto, Andrade (2015) realiza una colocación que resulta sumamente pertinente, el mismo expresa "[...] o trabalho doméstico, geralmente atribuído à mulher, assumiria um papel significativo para a conservação e reprodução da capacidade de trabalho" (ANDRADE, 2015, p. 281), además de agregar que los trabajos domésticos fornecen medios de subsistencia y asistencia a los miembros de la familia, garantizando las condiciones objetivas para la sobrevivencia de los futuros/as trabajadores.

Por otra parte, si sumamos el $14.3 \%$ de quienes consideran que las mujeres trabajan menos horas que los hombres, con el $42.9 \%$ que consideran que las mujeres trabajan la misma cantidad de hora que hombres, vemos como estas respuestas se contradicen por lo expuesto por Medeiros y Pinheiro, quienes mencionan "[...] mais mulheres trabalhando, e estas trabalhando por mais horas por semana. A combinação dos dois tipos de trabalho faz com que o tempo médio de trabalho total das mulheres seja superior ao dos homens" (MEDEIROS; PINHEIRO, 2018, p. 172). Cuando los autores hacen referencia a dos tipos de trabajo, refieren al trabajo pago, y al no pago. Demostrando de esta forma que el tiempo total de trabajo de las mujeres, es en media, superior al de los hombres.

Sobre esta misma línea de análisis, resultan sumamente pertinentes las indicaciones que realiza Andrade (2015), al traer a mención a Haug, quien expresa que la diferencia no es solamente entre producción y reproducción, sino mas bien entre vida y medios de vida, proponiendo mencionar que la reproducción es mas bien la subsistencia, expresando "[...] preferiría falar não de reproducão, mas sim de sustento de vida (subsistência) e desenvolvimento de atividades vitais" (HAUG, 2006 apud ANDRADE, 2015, p. 293).

Ahora bien, en cuanto a quien debería faltar al trabajo en caso de que su hijo/a se encuentre enfermo, el $86.7 \%$ responde "Necessariamente a mãe", lo que demuestra que se perciben las tareas de cuidado necesariamente como tareas realizadas por la mujer. De todas formas, el $100 \%$ de quienes respondieron manifestaron que no concuerdan con la afirmación de que "la mujer no debe trabajar fuera de casa", dejando demostrado de esta forma que se percibe la órbita publica como un espacio habitado también por la mujer.

Asimismo, bajo ese $86.7 \%$ que manifiesta que es necesariamente la madre quien se encarga de los cuidados resulta significante mencionar a Sorj (2014) quien expresa que el trabajo domestico continua siendo mayormente ocupado por mujeres. Esta categoría reúne características que indican su desvalorización social, tales como la baja remuneración, las largas jornadas de trabajo y su elevado nivel de informalidad. De esta forma, deja en claro como es que se trata de una ocupación situada entre las mas precarias ofrecidas por el mercado de trabajo. En estas trabajadoras se interseccionan categorías de clase, género y raza que a su vez hacen frente a dificultades para conciliar 
el trabajo doméstico remunerado con el trabajo doméstico no pago. Al tiempo que expresa;

\begin{abstract}
Para se entender o lugar estratégico que o trabalho doméstico remunerado assume no país é preciso posicioná-lo em relação a outras esferas de cuidado, como a repartição das tarefas domésticas entre homens e mulheres na família e as provisões públicas de educação infantil. Os debates sobre care têm frequentemente favorecido a análise das formas como se opera a sua externalização, seja para o mercado, seja para o Estado. A participação masculina na divisão sexual do trabalho doméstico, como uma reserva relevante de cuidado, permanece, até certo ponto, ausente das discussões, de tal forma que a questão do care aparece como um problema de e para as mulheres. (SORJ, 2014, p. 125).
\end{abstract}

De esta forma, se visualiza que, a pesar del progreso de las mujeres, sobre todo de aquellas que son madres, en el trabajo remunerado, ellas continúan asumiendo una parte desproporcional del trabajo doméstico y del cuidado (care). Resulta relevante también el presente fragmento de Sorj (2014), quien expresa; “[...] a identidade masculina continua a se reproduzir pela distância que os homens mantem e procuram preservar na esfera doméstica. A pouca dedicação do tempo não pago pelos homens contribui para a mercantilização do cuidado, o qual, por sua vez, assume um claro perfil de gênero" (SORJ, 2014, p. 126). Bajo este presupuesto, la autora demuestra cómo es que esta experiencia en el mercado de trabajo es distinta para hombres y para mujeres, y a su vez como es que esto impacta en la mercantilización del trabajo del cuidado, teniendo esto también un claro perfil de género en su mercantilización. Asimismo, cabe hacer mención que las sociedades afrontan diferencialmente las alternativas para el cuidado, las mismas pueden tomar el camino de la mercantilización, los servicios públicos, el trabajo no pago, prácticas cooperativas y colectivas, hasta redes de parientes, amigos/as, vecinos/as.

Prosiguiendo sobre el análisis, resulta relevante mencionar a Medeiros y Pinheiro, quienes identifican que "[...] o trabalho doméstico permanece feminino e que, embora contribuição relativa dos homens tenha aumentado, as mulheres fazem, ainda, pelo menos duas vezes mais trabalho doméstico do que eles" (MEDEIROS; PINHEIRO, 2018, p. 163).

De esta forma, y en concordancia con lo expuesto por Sorj (2014) anteriormente, se demuestra que el trabajo doméstico aún sigue siendo claramente ejecutado por las mujeres, mostrando como el tiempo femenino en la reproducción social es mayor que el masculino. Ambos autores además afirman que "Praticamente toda a população feminina adulta (88\%) realiza atividades de trabalho não pago. No entanto, apenas pouco menos de metade dos homens (46\%) emprega tempo em trabalho não pago" (MEDEIROS; PINHEIRO, 2018, p. 175).

En cuanto a la percepción de si se consideran las "actividades domésticas" como trabajo, también se obtuvo consenso en esto, siendo el $100 \%$ las respuestas que si consideran las actividades domesticas como trabajo. Respecto a que el hombre asuma tareas domésticas, el $100 \%$ expreso estar de acuerdo con ello. Resulta relevante traer a mención a Sorj, quien expresa "[...] o trabalho doméstico é, sem dúvida, um dos recursos responsáveis pela forte expansão do emprego de mulheres de classe média nas últimas décadas" (SORJ, 2014, p. 124). Es decir, al surgir el trabajo doméstico 
remunerado, fue uno de los motivos, directos o indirectos, de la inserción de mujeres en el mercado de trabajo. En ese diapasón, los datos revelan la opinión que los padres y madres poseen sobre quien debe poseer el principal ingreso familiar, el $86.7 \%$ declara que debe provenir "De ambos", mientras que el 13.3\% restante manifiesta que debe de "provenir del hombre".

Referente a si concuerdan con la siguiente afirmación "la igualdad de género es una forma de acabar con la discriminación y opresión contra las mujeres", el 76.9\% confirma concordar con esto, frente a un $23.1 \%$ que no concuerda. Asimismo, ante la siguiente afirmación "La igualdad de género es una forma de eliminar todas las formas de violencia contra las mujeres", un $69.2 \%$ manifiesta concordar con esto, frente a un $30.8 \%$ que no concuerda.

Resulta relevante ante la presencia de estos datos que plantean que el $76.9 \%$ de a quienes se les aplico el cuestionario concuerdan con que la igualdad de género es una forma de acabar con la discriminación y opresión de las mujeres, la necesidad de comprender a la "[...] opressão patriarcal como fenómeno universal e a-histórico" (ANDRADE, 2015, p. 286). Asimismo, para las mujeres su entrada al mercado de trabajo comprendió de forma permanente su conexión con el trabajo doméstico, para las mujeres, los límites temporales se duplican y multiplican entre trabajo doméstico y profesional, de esta forma "[...] opressão e exploração se acumulam e articulam, e por isso elas estão em situação de questionar a separação entre as esferas da vida -privada, asalariada, política- que regem oficialmente a sociedade moderna" (ANDRADE, 2015, p. 290).

De esta forma, visualizamos como la opresión se da como proceso asociado a la esfera privada, mientras que explotación se hace presente en la esfera pública. Además, cabe comprender que categorías dicotómicas tales como trabajo profesional y trabajo doméstico, producción y reproducción, explotación y opresión son categorías indisociables. Acrescentando sobre esto, resulta pertinente mencionar que la opresión de género es un fenómeno que apoya y sostiene a la sociedad ya que "a opressão da mulher constitui, portanto, inegavelmente, um dos pilares de sustentação da sociedade burguesa". (ANDRADE, 2015, p. 291).

De esta forma, visualizamos que la existencia de sectores oprimidos y marginalizados no es fruto de un acaso, sino que ello es resultado de un sistema que se hace presente mediante desigualdades, en una sociedad dividida en clases, y en un sistema económico que se sustenta en la explotación, y de esta forma "[...] a opressão cultural e social das mulheres é combinada á sua exploração económica, refrocando-se mutuamente" (ANDRADE, 2015, p. 291). De esta forma, se visualiza como el entrelazado de la explotación capitalista es una específica división de trabajo en relaciones de género históricas, demostrando que las diversas formas de opresión, que caracterizan a la sociedad capitalista, como las opresiones sexuales y raciales, están fuertemente respaldadas por las opresiones de género y, en consecuencia, la explotación de las mujeres. De esta forma, tanto la dominación patriarcal, como la dominación capitalista constituyen dos faces de un mismo modo de producir y reproducir la vida, colaborando para la manutención de determinado orden social.

Por último, en cuanto al papel de la escuela como institución que pueda trabajar en la promoción de esta igualdad de género, un 66.7\% plantea que "Si", que la escuela debe promover la igualdad de género, frente a un 33.3\% que plantea que "No". Además, en cuanto a si la escuela puede jugar un rol de promoción a erradicar las situaciones de 
violencia contra la mujer, un $69.2 \%$ manifiesta que "Si", frente a un $30.8 \%$ que plantea que "No."

Resulta relevante hacer mención a lo que Algebaile (2017) entiende sobre la principal preocupación del proyecto "Escolas sem Partido", ya que dicho proyecto muestra una inquietud con la presencia de discusiones relativas a cuestiones de género, orientación sexual y modelos familiares en el proceso formativo, lo que involucra directamente a las instituciones educativas, esta cuestión será retomada cuando se expongan las respuestas sobre el abordaje de la diversidad sexual en las escuelas a continuación en el presente artículo.

De todas formas, es pertinente visualizar como es que se comienza a comprender que la protección social de los niños y las niñas no excluye la participación de los padres en la educación de los hijos e hijas, siendo incentivada su participación en juntas escolares y en demás instituciones de enseñanza, en cuanto a esto Andrade (2015) plantea

(...) na medida em que a sociedade se responsabilizasse por propiciar todas as condições necessárias ao desenvolvimento mental, físico e psicológico das crianças - incluindo habitação, saúde, educação, arte, lazer -, país e filhos gozariam de mais tempo livre para o convívio e estabelecimento de uma relação de afeto e respeito" (ANDRADE, 2015, p. 278).

Ahora bien, en lo que respecta a la diversidad sexual, se planteo la siguiente afirmación a fin de indagar si se está de acuerdo con ella; "Concuerda con que los/as niños/as son influenciados a ser homosexuales si tienen contacto con Gays y Lesbianas o son informadas sobre diversidad sexual", siendo la respuesta a ello "No" representando un $76.9 \%$, frente a un $23.1 \%$ que manifiesta que "Si". La respuesta que se obtuvo ante esta afirmación resulta estar en consonancia con lo que afirma Rodrigo Ratier (2016), cuando menciona "Não há base em pesquisa para afirmar que a orientação sexual seja influenciável por alguém” (RATIER, 2016, p. 36). Además, el autor expone como es que diferentes historias de aprendizaje pueden dar como resultado dos padrones comportamentales similares. Es decir, observar comportamientos públicos similares entre dos individuos no significa necesariamente que el proceso de aprendizaje de esos padrones haya sido el mismo. De este modo, se sostiene que el primer punto a ser definido aquí es la importancia del estudio de la orientación sexual como un fenómeno complejo, constituido por diferentes padrones y funciones. Se expresa entonces que señalar que la orientación sexual es un fenómeno complejo implica, entre otras cosas, la necesidad de romper con la comprensión de la misma a través de los pares dicotómicos innato/aprendido. Asi, puede comprenderse que padrones comportamentales complejos son inexorablemente, efectos de la influencia de factores biológicos y culturales, estando estos en un ciclo continuo de modificaciones individuales. Y, por lo tanto,

(...) se pode considerar que a orientação sexual não seria resultante diretamente nem de fatores biológicos nem de culturais, mas que suas múltiplas formas de manifestação decorreriam de diferentes interações entre esses elementos e, consequentemente, de diferentes percursos de desenvolvimento ontogenético. (MENEZES; BRITO; HENRIQUES, 2010, p. 246). 
Por lo tanto, debemos entender que el género y la orientación sexual son fenómenos sociales complejos e interdependientes de la cultura, y no pueden definirse, a priori, en términos de influencias, determinaciones o incluso sanciones. De esta forma, la asunción de la existencia de la diversidad, expone de manera implícita la presencia de un referente (común) a partir del cual se define y señala la diversidad.

Diversidad es un término que plantea "variedad", "diferencia", lo que implica que hay un referente sobre el cual se marca la variación y la diferencia. Cuando hablamos entonces de diversidad sexual, estamos hablando de variedad sexual, de diferencia sexual, de lo que es diferente, distinto y variado con relación a algo establecido como "natural", "normal" o "común". (COLLIGNON GORIBAR, 2011, p. 136).

De esta forma, el reconomiento a la diversidad el cual se menciona en reiteradas oportunidades no contiene en su interior un principio moral sino un principio de referencia. Se hace necesario entonces reflexionar sobre la diversidad como una categoría analítica que no solo permite, son que obliga a demostrar el elemento constitutivo de la norma, y de lo considerado "normal", es decir, aquello que se encuentra dentro de los parámetros establecidos por la sociedad y es considerado deseable, sobre todo en lo que refiere a la sexualidad. Lo normal de esta forma, se hace presente a partir de lo que la mayoría de una sociedad hace, piensa, define y habita como deseable/bueno, y de esta forma lo anormal queda designado a aquello que hacen, piensan y determinan las minorías.

Respecto a su opinión sobre la diversidad sexual, resulta relevante traer la siguiente grafica que demuestra Índice de indiferencia numeroso que nos lleva a pensar que la falta de interés en el tema puede proporcionar obstáculos para una mejor aceptación de la diversidad sexual.

Gráfica 2 - Opinión, por parte de los padres y madres de los alumnos, sobre la diversidad sexual

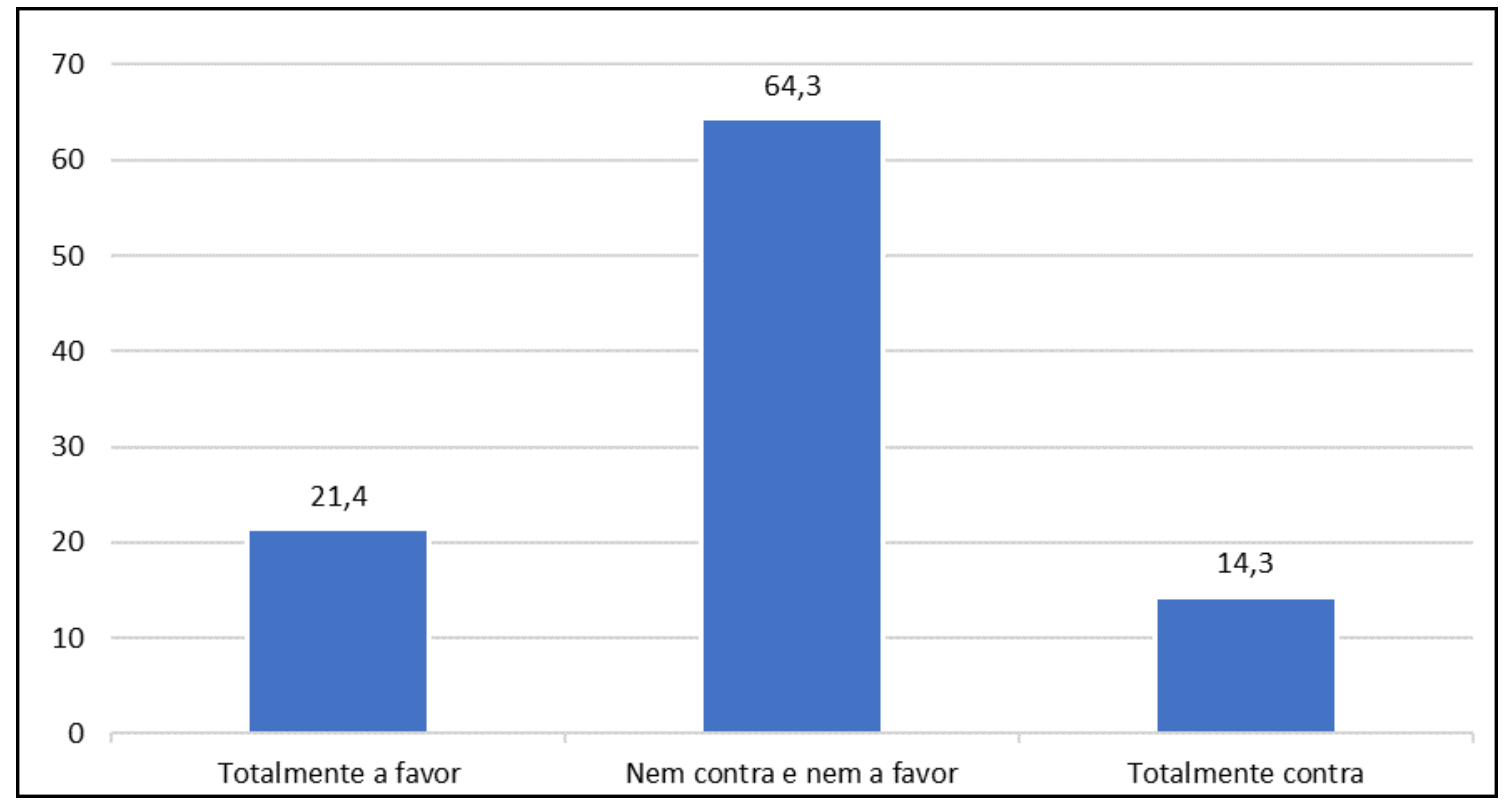

Fonte da pesquisa dos autores. 
En cuanto al estar de acuerdo con la existencia de personas homosexuales, un $57.1 \%$ declaro estar "totalmente a favor", un $28.6 \%$ manifiesta "nem contra e nem a favor, sou indiferente", y un 14.3\% plantea "totalmente contra". En lo que refiere a si el preconcepto contra las personas homosexuales debe ser combatido, un $85.7 \%$ plantea que si debe ser combatido, frente a un $14.3 \%$ que expresa que no debe ser combatido.

Observamos que la existencia de opiniones neutrales (ni a favor ni en contra) demuestra tendencias a trivializar los problemas sociales resultantes de la opresión de género y sexualidad. Con respuestas evasivas sobre el tema, los padres y las madres de los estudiantes están más cerca de las opiniones mayoritarias que tienden a reproducir las desigualdades. De esta manera, existe el riesgo de naturalizar los diversos procesos de opresión que marcan profundamente a la sociedad porque no permite una discusión crítica densa y permanente.

Respecto a su opinión sobre si la escuela debe de trabajar para el reconocimiento de la diversidad sexual, un $64.3 \%$ expresa que la escuela "Si" debe de trabajar en ello, frente a un $35.7 \%$ que manifiesta que "No". A fin de poder comprender esta respuesta que hace alusión al rol de la escuela en el abordaje para el reconocimiento de la diversidad sexual, es relevante hacer referencia al proyecto "Escola sem Partido" que claramente incide en la percepción sobre ello.

Es importante mencionar que el proyecto "Escola sem Partido" se trata de un proyecto que plantea "[...] a retirada do pensamento crítico, da problematização e da possibilidade de se democratizar a escola" (MANHAS, 2016, p. 16). Dicho proyecto propone como uno de sus ejes de que las escuelas trabajan "convicciones morales y valores" que no corresponden con los pensamientos de las familias, quienes son los verdaderos responsables por esta "parcela" de la educación, y no las instituciones de enseñanza.

Además, este proyecto de "Escolas sem Partido", tal como expresa Algebaile, 2017, "[...] mostra uma nítida preocupação com a presença, no processo formativo, de discussões que problematizem as concepções políticas, socioculturais e económicas hegemónicas, especialmente as relativas ás questões de gênero, orientação sexual e modelos familiares" (ALGEBAILE, 2017, p. 67). Asimismo, se resalta la importancia de debatir cuestiones de género y sexualidad en las escuelas, para que estas diferencias no sean luego transformadas en desigualdades.

Resulta sumamente pertinente traer a colación a Salomão Ximenes (2016) quien expresa:

\footnotetext{
Nesse sentido, preservar o direito dos país nos âmbitos informal e não-formal não é incompatível com o tratamento de questões morais, políticas e sexuais na educação formal pública, mas o contrário, já que a inclusão de tais temas no currículo é parte do direto dos estudantes de ver ampliados os seus referenciais a partir de concepções diversas, republicanas e científicas, todas necessárias ao pleno exercício da autonomia individual e da cidadania (XIMENES, 2016, p. 55, 56).
}

Asimismo, la necesidad de abordar la temática de género y diversidad sexual en las escuelas está en relación con la propia idea de educación, entendiendo que la educación es la apropiación de la cultura, y comprendiendo que la "(...) cultura é tudo aquilo que a humanidade produziu e produz" (CARA, 2016, p. 46). De esta forma, el 
derecho a la educación es el derecho de que todos/as puedan apropiarse de la cultura, tornándose sujetos autónomos, capaces de comprender y participar del mundo, aprendiendo todo lo que es posible y necesario para la realización de la vida, de esta forma "A escola, portanto, não ensina apenas conhecimentos, mas também valores, formas de agir, ser e estar no mundo" (CARA, 2016, p. 46).

Prosiguiendo sobre las respuestas al cuestionario, en lo que refiere al sentimiento con lidiar con personas homosexuales, los/as encuestados/as comentan emociones agradables, tales como "Empatia" (30.8\%), "Alegria" (15.4\%), "Satisfação" (7.7\%), "Conforto" (7.7\%), frente a un 30.8\% que expresa "Nao sinto nada, sou indiferente", y un $7.7 \%$ que expreso "Aversão".

Gráfica 3 - Sentimientos sobre la diversidad sexual

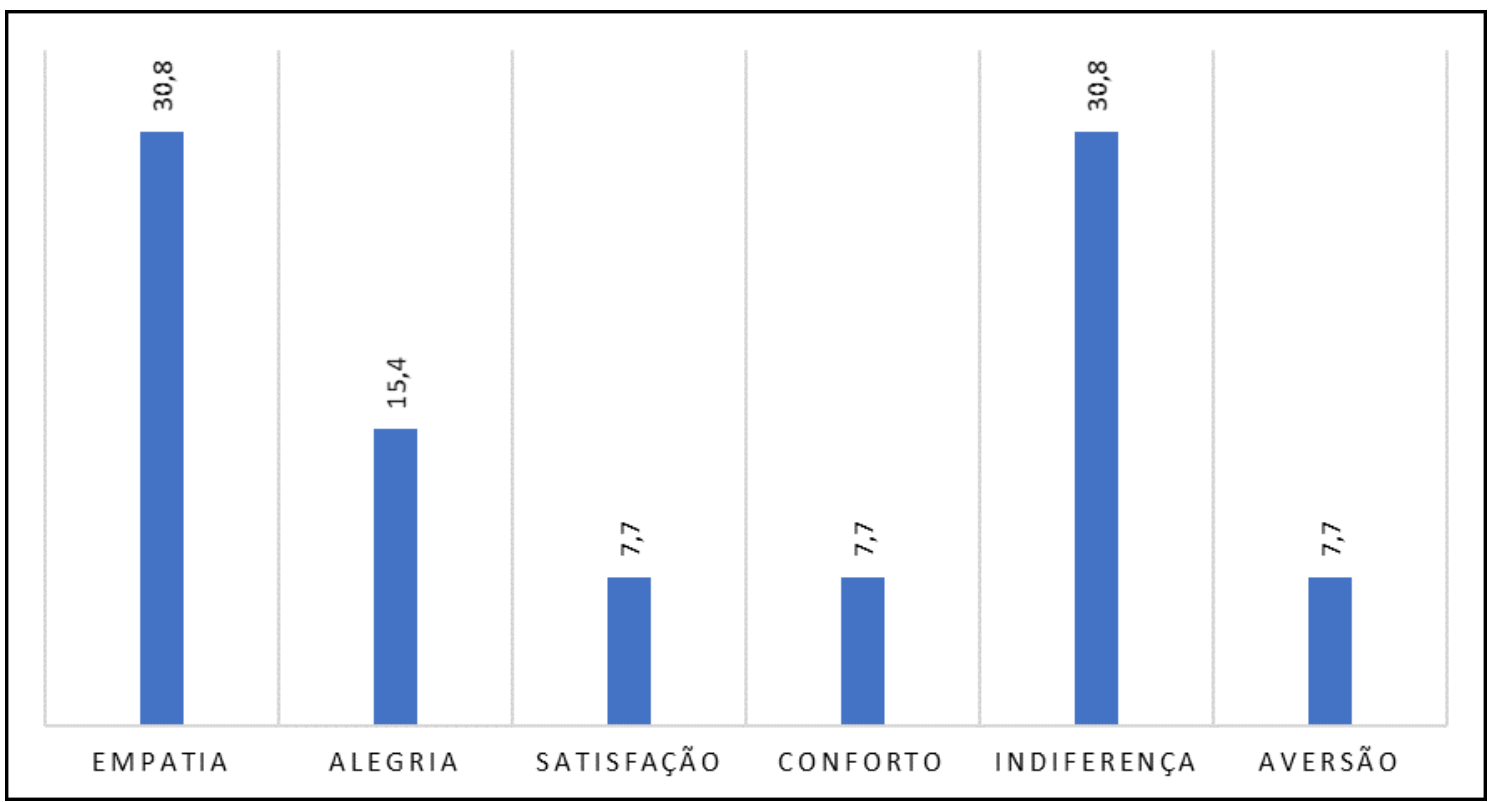

Fonte da pesquisa dos autores.

En cuanto a si se considera a la escuela como un espacio donde se de la existencia de personas homosexuales, un $63.5 \%$ expresa que "Si" es un espacio, frente a un $36.4 \%$ que considera que "No". Asimismo, en cuanto a si se considera a la escuela como un espacio para la existencia de transexuales, un 54.5\% expresa que "Si", frente a un $45.5 \%$ que expresa que "No".

Las opiniones, destacadas aquí, pueden resaltar en el plano de las relaciones privadas, el vínculo de los encuestados con la falta de compromiso en relación con los problemas sociales y los problemas relacionados con la opresión de género y sexualidad, además podemos notar que la indiferencia se caracteriza por la falta de empatizar unos con otros.

En estas preguntas anteriores sobre el rol de la escuela en el trabajo para el reconocimiento de la diversidad sexual, y como institución en la que se hagan presentes personas homosexuales y transexuales resulta relevante traer a mención a Manhas quien expresa; "[...] uma das razões do abandono escolar é a discriminação relativa ao 
publico LGBTI. Motivações mais do que suficientes para discutirmos gênero nas escolas" (MANHAS, 2016, p. 17).

Desde esta forma visualizamos la importancia de abordar la temática de género y sexualidad en la institución educativa, debido a que una de las razones de abandono escolar es la discriminación que sufren las identidades pertenecientes a la comunidad LGBTI, y ello se vio reflejado en las dos respuestas anteriores, sobre todo en las que hacen referencia a la presencia de personas homosexuales en la escuela, en la que el porcentaje de estar en contra a esto fue de $36.4 \%$, y este porcentaje se eleva a $45.5 \%$ cuando la pregunta se direcciona a la presencia de personas transexuales en la escuela.

\section{CONSIDERACIONES FINALES}

Durante la presentación de datos, mediante el uso de gráficas y porcentajes, además de su posterior análisis sustentado en diferentes referencias teóricas que contemplan y generan un interesante análisis sobre el contexto socio-histórico en el cual se habita y se generan significaciones/percepciones es que se ha ido construyendo el presente artículo.

Ahora bien, presentando un análisis sobre los datos presentados y de manera general, en cuanto a la igualdad de género se puede señalar que la cantidad de horas trabajadas por las mujeres es claramente superior a la de los hombres, aumentando esta cantidad de horas para el caso de aquellas mujeres madres. Asimismo, la clara existencia entre la combinación entre trabajo profesional y trabajo doméstico no pago. Conjunto a esto, el surgimiento del trabajo doméstico remunerado, que posee un claro perfil de género, raza y clase social como categorías indisolubles. Además, la necesidad de comprender la función del trabajo doméstico como generador de medios de subsistencia y asistencia a los miembros de la familia. Surge la necesidad de adoptar el término "sustento de vida" o "desarrollo de las actividades vitales" en detrimento del término "reproducción". Prosiguiendo, se hace claro el énfasis en señalar la desvalorización social que posee el trabajo doméstico, lo que se expresa en su precarización.

Además, se señala el rol predominante de las mujeres en las tareas de cuidado (care), generándose como efecto de esto la mercantilización de dichas tareas. Por último, se hace necesaria la indicación de comprender la existencia conjunta entre opresión/explotación, fenómenos que se articulan y acumulan, cuestionándose su separación para la realización de un análisis en el ámbito privado-asalariado-político e las actuales sociedades modernas.

En lo que refiere a la diversidad sexual, las principales consideraciones resultaron ser la comprensión de que la orientación sexual no resulta ser influenciada por nadie, sino que ello va de la mano con entender que diferentes trayectos históricos de aprendizajes pueden acabar en padrones comportamentales similares. De esta forma, se hace necesario el abordaje de la orientación sexual como fenómeno complejo, lo que supone la ruptura con la idea de comprender a la misma a partir del uso del par dicotómico innato/aprendido. Asimismo, se señala la existencia de la diversidad como origen en cierto "referente común", considerado "normal", establecido por parámetros previamente existentes sobre aquello que es entendido deseable. Además, resulto necesario recurrir a la interferencia del proyecto "Escola sem Partido" como proyecto que propone la retirada del pensamiento crítico-problematizador en la escuela, 
interfiriendo en cuestiones de discusiones que trabajen la temática género, orientación sexual, identidades y configuraciones de modelos familiares.

Resulta sumamente pertinente para finalizar realizar una articulación entre igualdad de género y diversidad sexual, visualizando como la influencia de los medios de comunicación generan y modifican estas percepciones sobre las temáticas. En lo que refiere a la igualdad de género, se puede visualizar que las respuestas no fueron tan conservadoras como si lo fueron en la temática referente a la diversidad sexual. En la igualdad de género, las personas comentan que han escuchado hablar sobre ello en "jornal falado" (60\%), mientras que en la diversidad sexual ese porcentaje baja para $35.7 \%$, lo que demuestra que los medios de comunicación, han contribuido en hacer que esta percepción sobre la igualdad de género sea menos conservadora que las percepciones sobre la diversidad sexual, señalando que han utilizado estrategias tales como colocar mujeres para dirigir el informativo, el abordaje de la temática de violencia y misoginia en diversas novelas, o reportajes periodísticos que exponen sobre la temática de los servicios domésticos, ingresos de mujeres y de hombres desiguales.

He de allí que surge la importancia de comprender a la economía y a la ideología como componentes relacionados en un único sistema de determinación. De esta forma y de modo genérico ideología es un término utilizado para los procesos por el cual el significado es producido, desafiado, reproducido y transformado; "Como o significado é negociado primordialmente através dos meios de comunicação e significação, é possível sugerir que a produção cultural proporciona um importante lugar para a construção de processos ideológicos" (BARRETT, 1984 apud ANDRADE, 2015, p. 288). De esta forma, estas percepciones que son "producidas, desafiadas, reproducidas y transformadas" a través de la influencia de los medios de comunicación sugieren una producción cultural en la construcción de estos procesos ideológicos.

Finalmente, resulta pertinente hacer mención a comprender que la escuela cumple un rol en la apropiación de la cultura, no enseñando únicamente conocimientos, sino también valores, formas de actuar, ser, y estar en el mundo. Además, la existencia del abandono escolar como fenómeno que se da por razones de discriminación resulta una motivación más que suficiente para discutir esta temática en el ámbito educativo con los propios sujetos de la educación. De esta forma, el abordaje de la diversidad sexual, y la igualdad de género, y su importancia de generación de conocimientos e investigaciones sobre ello en la esfera educacional resulta relevante a la hora de generar estrategias para reducir los casos de violencia, opresión, y LGBTfobia.

\section{REFERENCIAS}

ALGEBAILE, Evaline. Escola sem partido: o que é, como age, para que serve. In: FRIGOTTO, Gaudencio. Escola "sem" partido: esfinge que ameaça a educação e a sociedade brasileira. Rio de Janeiro: UERJ, LPP, 2017, p. 63-64.

ANDRADE, Joana El-Jaick. O feminismo marxista e a demanda pela socialização do trabalho doméstico e do cuidado com as crianças. Rev. Bras. Ciênc. Polít., Brasília, n. 18, p. 265-300, dez. 2015. Disponível em: <http://www.scielo.br/scielo.php?script=sci_arttext\&pid=S0103- 
$33522015000400265 \& \operatorname{lng}=$ pt\&nrm=iso $>$.

Acesso

em 13 abr.

2020. https://doi.org/10.1590/0103-335220151810.

CARA, Daniel. O programa "Escola sem Partido" quer uma escola sem educação. In: MALTA, Maria Machado; VIEIRA, Cleib Aparecida, PRAZERES, Michelle. A Ideologia do movimento Escola Sem Partido: 20 autores desmontam o discurso. São Paulo: Ação Educativa, 2016, p. 43-48.

COLLIGNON GORIBAR, María Martha. Discursos sociales sobre la sexualidad: narrativas sobre la diversidad sexual y prácticas de resistencia. Comun. soc, Guadalajara, n. 16, p. 133-160, dic. 2011. Disponible en <http://www.scielo.org.mx/scielo.php?script=sci_arttext\&pid=S0188252X2011000200006\&lng=es\&nrm=iso>. Acesso em 13 abr. 2020.

MANHAS, Cleomar. Nada mais ideológico que "Escola sem Partido". In: MALTA, Maria Machado; VIEIRA, Cleib Aparecida, PRAZERES, Michelle. A ideologia do movimento escola sem partido: 20 autores desmontam o discurso. São Paulo: Ação Educativa, 2016, p. 15. 22.

MEDEIROS, Marcelo; PINHEIRO, Luana Simões. Desigualdades de gênero em tempo de trabalho pago e não pago no Brasil, 2013. Soc. Estado., Brasília, v. 33, n. 1, p. 159185, abr. 2018. Disponível em $<$ http://www.scielo.br/scielo.php?script=sci_arttext\&pid=S010269922018000100159\&lng=pt\&nrm=iso>. Acesso em 13 abr. 2020. https://doi.org/10.1590/s0102-699220183301007.

MENEZES, Aline Beckmann; BRITO, Regina Célia Souza; HENRIQUES, Alda Loureiro. Relação entre gênero e orientação sexual a partir da perspectiva evolucionista. Psic.: Teor. e Pesq., Brasília, v. 26, n. 2, p. 245-252, June 2010. Available from $<$ http://www.scielo.br/scielo.php?script=sci_arttext\&pid=S010237722010000200006\&lng=en\&nrm=iso>. Access on 13 Apr. 2020. https://doi.org/10.1590/S0102-37722010000200006.

RATIER, Rodrigo. 14 perguntas e respostas sobre o "Escola sem Partido". In: MALTA, Maria Machado; VIEIRA, Cleib Aparecida, PRAZERES, Michelle. A Ideologia do movimento Escola Sem Partido: 20 autores desmontam o discurso. São Paulo: Ação Educativa, 2016, p. 29-42.

SORJ, Bila. Socialização do cuidado e desigualdades sociais. Tempo soc., Sao Paulo, v. 26, n. 1, p. 123-128, jun. 2014. Disponible en: $<$ http://www.scielo.br/scielo.php?script=sci_arttext\&pid=S010320702014000100009\&lng=pt\&nrm=iso>. Acceso em 13 abr. 2020. https://doi.org/10.1590/S0103-20702014000100009. 
XIMENES, Salomão. O que o direito a educação tem a dizer sobre "Escola sem Partido"?. In: MALTA, Maria Machado; VIEIRA, Cleib Aparecida, PRAZERES, Michelle. A ideologia do movimento escola sem partido: 20 autores desmontam o discurso. São Paulo: Ação Educativa, 2016, p. 49-58.

Recebido:06/05/2020

Aprovado: 10/07/2020 